\title{
O pulso de inundação no sistema de baías Caiçara, Norte do Pantanal Mato-Grossense (Brasil), determina a reprodução de Acestrorhynchus pantaneiro (Menezes, 1992) (Characiformes, Acestrorhynchidae)
}

\author{
The flood pulse as a reproduction descriptor of the Acestrorhynchus \\ Pantaneiro (Menezes, 1992) (Characiformes, Acestrorhynchidae) in \\ the Caiçara lake system, northern Pantanal, Alto Paraguay
}

Karine Marreiro Soares ${ }^{[a]}$, Claumir Cesar Muniz ${ }^{[b]}$, Adonias Barbosa de Castro[ ${ }^{[c]}$, Ernandes Sobreira Oliveira Junior ${ }^{[d]}$

[a] Acadêmica do Curso de Ciências Biológicas, Universidade do Estado de Mato Grosso (UNEMAT), Cáceres, MT - Brasil, e-mail: karinemarreiro@hotmail.com

[b] Biólogo, Doutor em Ecologia e Recursos Naturais, Professor adjunto da Universidade do Estado de Mato Grosso (UNEMAT), Laboratório de Ictiologia do Pantanal Norte, Centro de Pesquisa em Limnologia, Biodiversidade e Etnobiologia do Pantanal (CELBE), Cáceres, MT - Brasil, e-mail: claumir@unemat.br

[c] Biólogo, Laboratório de Ictiologia do Pantanal Norte, Centro de Pesquisa em Limnologia, Biodiversidade e Etnobiologia do Pantanal (CELBE), Universidade do Estado de Mato Grosso (UNEMAT), Cáceres, MT - Brasil, e-mail: adoniasdecastro@hotmail.com

[d] Biólogo, Mestre em Ecologia e Conservação da Biodiversidade, Laboratório de Ictiologia do Pantanal Norte, Centro de Pesquisa em Limnologia, Biodiversidade e Etnobiologia do Pantanal (CELBE), Universidade do Estado de Mato Grosso (UNEMAT), Cáceres, MT - Brasil, e-mail: ernandes@unemat.br

\section{Resumo}

O objetivo do estudo foi analisar o aspecto reprodutivo do Acestrorrinchus pantaneiro (peixe comum no Pantanal Mato-Grossense), correlacionando-o com o pulso de inundação (sistema de Baías Caiçara, Norte do Pantanal MatoGrossense, no alto Paraguai), fator determinante à manutenção do pantanal e sua biodiversidade. Os peixes foram capturados, bimestralmente, de setembro de 2005 a setembro de 2007, em períodos diurnos e noturnos em seis pontos do sistema. Para a coleta dos espécimes em área aberta, foram utilizadas redes de arrasto e tarrafas, de alturas, tamanhos e malhas variadas. Na coleta dos peixes associados às macrófitas, utilizou-se tela de material plástico, tipo mosquiteiro, armada em estrutura metálica. Após a coleta, os espécimes foram fixados em formalina a 4\% e armazenados no Laboratório de Ictiologia da Universidade do Estado de Mato Grosso (UNEMAT), onde foram obtidos os dados biométricos, determinando-se o sexo e a fase gonadal. Dos 867 espécimes de A. pantaneiro coletados, 48,6\% eram fêmeas, 38,6\% machos e 12,9\% não foi possível determinar o sexo. 0 estádio de desenvolvimento gonadal em repouso predominou nos espécimes coletados nos períodos de vazante e estiagem. Já os períodos de enchente e cheia foram marcados pelo predomínio de indivíduos em maturação e maturos, além da presença de indivíduos imaturos, juvenis. A desova dos peixes destes sistemas de baías ocorre com mais intensidade no final do período de enchente. Constatou-se que o pulso de inundação atua como um fator determinante da reprodução de A. pantaneiro, uma vez que a espécie estudada mostrou preferências em se reproduzir no período de águas altas.

Palavras-chave: Dinâmica hidrológica. Estado gonadal. Planície inundável. Peixe. Pantanal Mato Grossense. 


\section{Abstract}

This study aimed to analyze the flood pulse as a descriptor of the Acestrorhynchus pantaneiro reproduction in the Caiçara Lake system, in the northern portion of the Mato Grosso Pantanal, Alto Paraguay. The fishes were caught bimonthly from September 2005 to September 2007, in daytime and nighttime periods at six points in the system. To collect specimens in open area, it were used trawl nets and cast nets from heights, sizes and different meshes. In the collection of fish associated with macrophytes, it was used a plastic screen, type fly armed on metal frame. After collecting the specimens, they were fixed in 4\% formalin and stored in the Laboratory of Ichthyology, University State of Mato Grosso (UNEMAT), where the biometric data were obtained, as well as the sex and gonadal stage were determined. Of the $867 \mathrm{~A}$. pantaneiro specimens collected, $48.6 \%$ were females, $38.6 \%$ males and $12.9 \%$ could not identified the sex. The gonadal development home predominated in specimens collected during periods of low water and drought; however, flood periods were marked by the predominance of maturing and mature individuals, and the presence of immature juveniles. The fish spawning lakes of these systems occurs with more intensity during the end of the flood. Thus, we find that the flood pulse acts as a descriptor of the reproduction of A. pantaneiro in the Caiçara lakes system, since the studied species showed preferences to reproduce in the high-water period.

Keywords: Hydrologic dynamics. Gonadal stage. Wetland. Fish. Pantanal Mato Grossense.

\section{Introdução}

O Pantanal mato-grossense é a maior planície inundável do planeta (Brasil, 1979), dotada de uma vasta área alagável, regulada pelo pulso de inundação anual (Junk e da Silva, 1996), e marcada por períodos distintos de cheia e seca, que promovem inúmeras alterações, marcantes nos habitats aquáticos e terrestres (Da Silva et al., 2001).

0 pulso de inundação no Pantanal é responsável por promover alterações na disponibilidade de habitats aquáticos, influenciando a abundância de algumas espécies, devido a variação dos eventos anuais de enchente e estiagem. A quantidade de alimentos dispostos e as relações tróficas mantidas entre as espécies podem sofrer variações drásticas, principalmente quando a ausência de conectividade hidrológica é mais pronunciada (Da Silva et al., 2001).

0 pulso de inundação forma baías que, no período de estiagem, permanecem ou não conectados ao rio, e essa dinâmica permite as interações entre fatores bióticos e abióticos (Muniz, 2010). 0 período de extravasamento das águas nas margens do canal principal dos rios da planície de inundação permite que os peixes explorem estas áreas alagadas em busca de alimento e abrigo para reprodução (Rodríguez e Lewis, 1994)

Estudos envolvendo a reprodução de peixes são de grande importância, visto que este é um dos principais processos durante o seu ciclo de vida e na sua dinâmica populacional. Os recursos explorados no ambiente pelos indivíduos originados no período reprodutivo são importantes para o sucesso da sua população (Wotton, 1984).

Os aspectos reprodutivos dos peixes, baseados na investigação gonadal, vêm sendo utilizados para compreender o papel ecológico das espécies em um ambiente aquático. Nas fases seguintes do desenvolvimento das gônadas, há um aumento no seu volume e no peso dos ovários. Dessa forma, o índice gonadal é um indicador quantitativo para avaliar o período de reprodução da espécie em estudo (Vazzoler, 1996).

Acestrorhynchus pantaneiro é um peixe de água doce, popularmente conhecido como peixe-cachorro, encontrado em abundância em lagos e lagoas do Pantanal mato-grossense (Britsk et al., 2007) e está incluído na ordem Characiformes, família Acestrorhynchidae (Buckup et al., 2007). 0 gênero Acestrorhynchus é representado por 17 espécies que ocupam diversos habitats, sendo mais comuns nos ambientes lênticos, sendo sua distribuição específica da América do Sul (Saccol-Pereira et al., 2006).

A espécie A. pantaneiro é um peixe de corpo alongado, alcançando em média $30 \mathrm{~cm}$ de comprimento. Possui uma mancha redonda umeral característica (Menezes, 1992) e hábito alimentar carnívoro, basicamente ictiófago, podendo vir a complementar 
a sua dieta com crustáceos (Zaniboni-Filho et al., 2004), insetos (Britski et al., 2007), bem como também se alimentar da própria espécie, inferindo a possibilidade de canibalismo (Novaes et al., 2004).

Não são muitos os estudos dirigidos às espécies de pequeno porte, especialmente as espécies sem grande potencial comercial. Porém, a abundância de A. pantaneiro em baías do Pantanal mato-grossense faz com que as comunidades ribeirinhas as utilizem como fonte de complemento alimentar, em virtude de representar, junto a outras espécies de peixes, um dos meios de obtenção de proteína animal (Nogueira e Oliveira, 2000). O A. pantaneiro pode contribuir com o manejo ecossistêmico e como fonte alternativa à pesca no Pantanal.

0 presente estudo teve como objetivo analisar a importância do pulso de inundação como descritor da reprodução de A. pantaneiro em um sistema de baías do Pantanal Norte.

\section{Material e método}

\section{Área de estudo}

0 presente trabalho foi realizado em um conjunto de baías, localizadas entre as coordenadas $16^{\circ}$

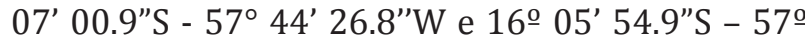
45' 22.3”W, distando 8,0 km da cidade de Cáceres, Pantanal Norte (MT) (Figura 1). Este conjunto de baías é denominado Sistema de Baías Caiçara, em referência a localidade onde são encontradas, na margem direita do Rio Paraguai.

A planície pantaneira apresenta pluviosidade elevada, marcada por períodos de grandes cheias e estiagens (Junk e Da Silva, 1996), com precipitação média de $1.599 \mathrm{~mm}$ e temperatura média de $24{ }^{\circ} \mathrm{C}$, com a mínima de $11{ }^{\circ} \mathrm{C}$ e máxima de $42{ }^{\circ} \mathrm{C}$ (Ferreira, 1997). 0 clima da região, de acordo com a

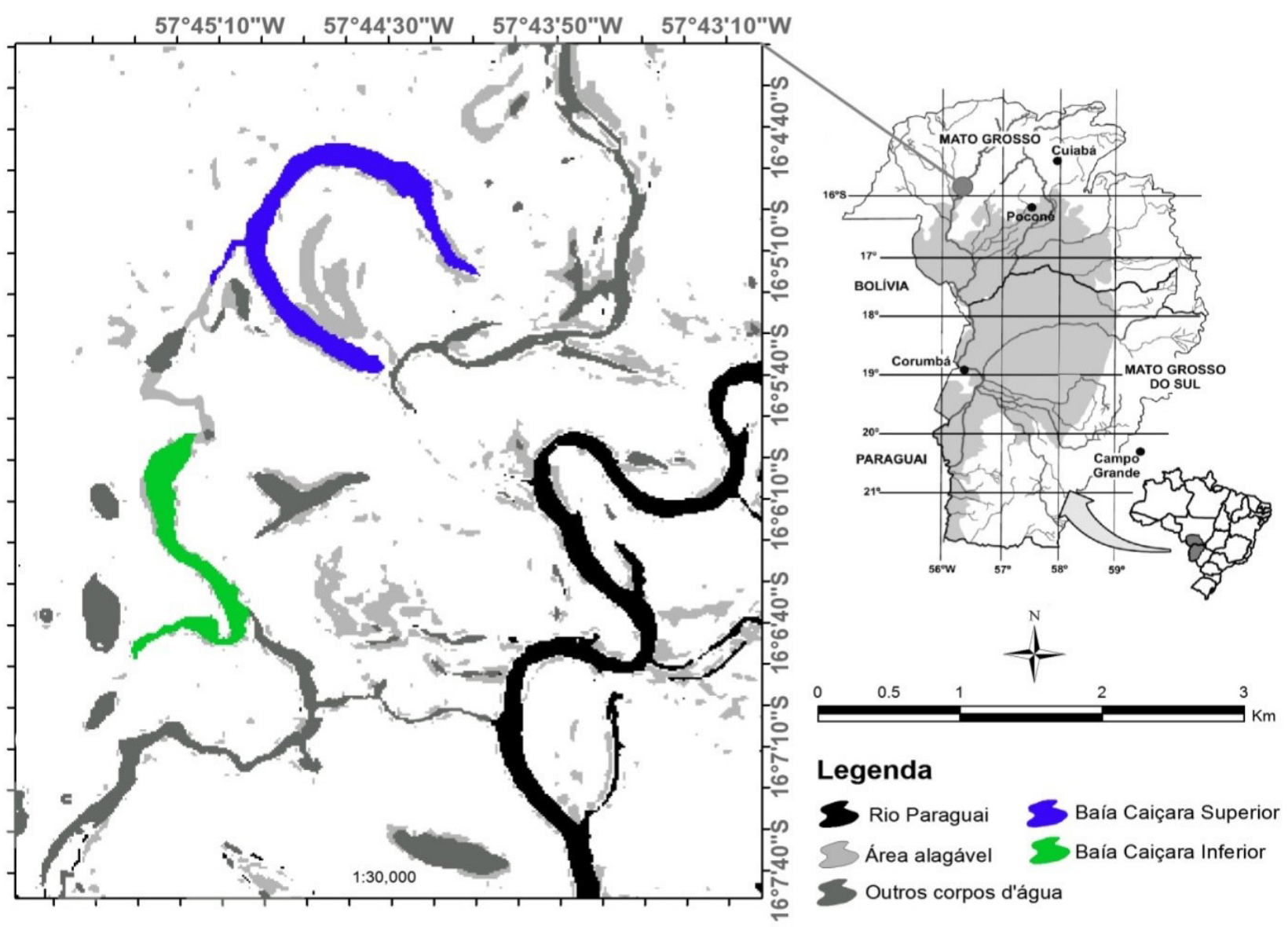

Figura 1 - Área de estudo na Planície de Inundação do rio Paraguai, no Sistema Baía Caiçara 
classificação de Köppen, é tropical de savana (AW) (Vianello e Alves, 2000).

No período chuvoso, o sistema de baías Caiçara constitui um corpo de água único, com características semi-lóticas, conectado lateralmente ao rio Paraguai e a outras baías. Durante a estiagem (período de seca), o sistema Caiçara apresenta dois subsistemas, classificados como Baía Caiçara Superior (BCS) e Baía Caiçara Inferior (BCI). Esses subsistemas são distintos com diferentes graus de conectividade lateral com o rio Paraguai, o que lhes confere características hidrodinâmicas diferenciadas, onde $\mathrm{BCI}$, mesmo nos períodos de estiagem, mantém conectividade com o canal principal do rio e BCS perde a conexão com o canal principal no período de seca (Muniz, 2010).

\section{Coleta e análise dos dados}

O estudo foi realizado com exemplares de espécimes de A. pantaneiro coletados, bimestralmente, no período de setembro de 2005 a setembro de 2007, em períodos diurnos e noturnos. Foram amostrados três pontos equidistantes na BCS e BCI, respectivamente. A distância entre as duas baías é de 1,5 km.

Dados ambientais como temperatura e profundidade foram tomados em campo com auxílio de uma sonda Hach multiparamétrica e do disco de Secchi, respectivamente.

Para a captura de peixes associados às macrófitas aquáticas, foi utilizada tela de material plástico, tipo mosquiteiro (malhas 2, 3, 4, 5 e $6 \mathrm{~cm}$ de entrenós), armada em estrutura metálica com $105 \mathrm{~cm} \mathrm{x}$ $205 \mathrm{~cm} \times 100 \mathrm{~cm}$ nos bancos de macrófitas (tréplicas) durante 2 horas. Os peixes de áreas abertas foram capturados com rede de arrasto (malhas de 2, $3,4 \mathrm{~cm}$ entrenós com dimensões de $35 \mathrm{~m} \mathrm{x} \mathrm{2,2} \mathrm{m).}$ Para a ictiofauna de ambientes mais profundos, nos períodos de cheia, foi utilizado tarrafas de diferentes malhas e alturas (tarrafas de malhas com $2 \mathrm{~cm} \mathrm{e}$ $3 \mathrm{~cm}$ nas áreas de meandros).

Os espécimes foram capturados, fixados em formalina a 4\%, e transportados para o laboratório de Zoologia, onde foram identificados e armazenados no Laboratório de Ictiologia da Universidade do Estado de Mato Grosso (UNEMAT).

Os dados biométricos (peso, comprimento padrão e total) foram obtidos por meio de um paquímetro; a mensuração do peso gonadal foi feito por meio de balança de precisão e o IGS tomado de acordo com Vazzoler (2006). A identificação do sexo foi realizada pela observação macroscópica das gônadas.

Após a tabulação dos dados, foram obtidos os estádios reprodutivos, de acordo com a maturação das gônadas, sendo classificados como: imaturos, maturação, maturos, esvaziados e repouso, de acordo com o método de Vazzoler (2006).

\section{Resultados e discussão}

Foram coletados 865 espécimes de A. pantaneiro no sistema de baías Caiçara. Destes, $48,6 \%$ eram fêmeas, $38,6 \%$ machos e $12,9 \%$ não determinados. Houve predominância de fêmeas, dado este também evidenciado por Guerra et al. (2009), em trabalho realizado em um reservatório com espécie do mesmo gênero, onde o número de fêmeas foi superior. Krinski (2010) relata também que, no pantanal de Poconé (MT), em estudo com esta espécie, houve maior número de fêmeas. Wootton et al. (1978) relatam que alguns fatores de pressão ambiental, como cheias e secas prolongadas influenciam de maneira positiva ou negativa a proporção de machos e fêmeas em um ambiente.

A. pantaneiro, neste sistema de baías pertencentes ao Pantanal, apresentou-se em maturação a partir de 9,7 cm para machos e $11,9 \mathrm{~cm}$ para fêmeas. 0 comprimento padrão (CP) variou de 2,8 a $27 \mathrm{~cm}$; o comprimento total (CT) variou de 3 a $29 \mathrm{~cm}$; enquanto que o peso $(\mathrm{P})$ variou de 0,32 a 338,35g.

Para o sistema de baías Caiçara, estas diferenças estão relacionadas à metodologia utilizada, uma vez que, no trabalho de Krinski (2010), os apetrechos eram mais seletivos, quando comparados com tela e rede de arrasto. A área de margem das baías serve de proteção para os indivíduos jovens, com vegetação e "locas", dificultando assim a captura destes espécimes (Paiva, 1974). Neste trabalho, o peso de A. pantaneiro pode ser influenciado pelas dimensões dos indivíduos, que, em média, foi maior do que aqueles encontrados por Paiva (1974)

Nos períodos de vazante e estiagem, indivíduos em fase de repouso reprodutivo foram predominantes (Figura 02), isto significa que esta espécie não se reproduz nestas estações do ano. Ao se considerar os indivíduos em maturação, esta variação foi inversa, sendo maior no período de enchente. 


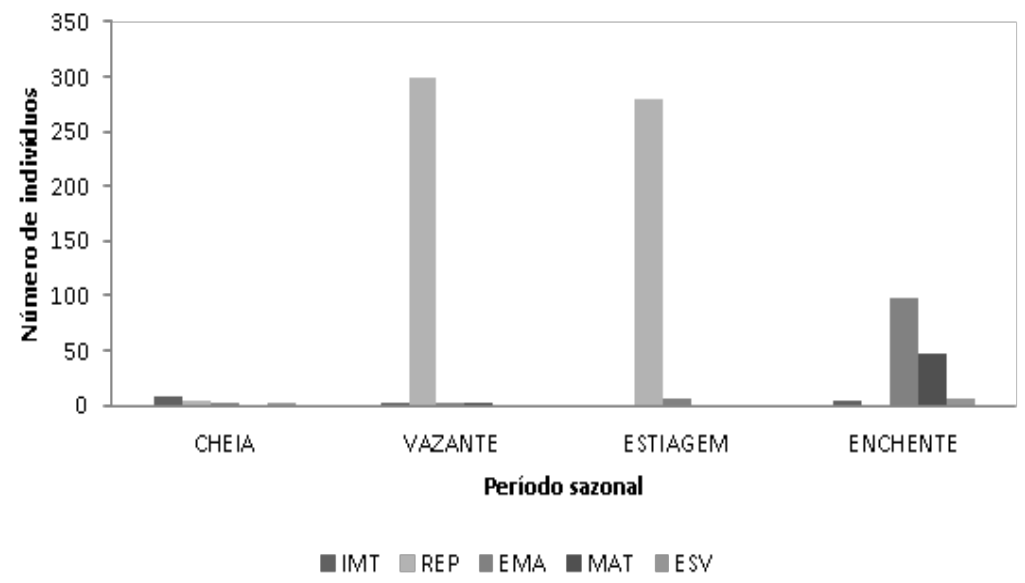

Figura 2 - Números de indivíduos em seus estágios de desenvolvimento gonadal de Acestrorhynchus pantaneiro no Sistema de baías Caiçara, Pantanal Norte (MT) (2005/2007).

Fatores ambientais, fisicos, químicos e disponibilidade de alimento podem influenciar o processo reprodutivo da fauna ictiológica (Ribeiro, 2012). Estudos realizados no sistema de planície de inundação do alto rio Paraná evidenciaram que a estrutura e o funcionamento deste sistema são controlados pelas inúmeras variações do nivel hidrológico (Agostinho e Gomes, 1997).

Segundo Wootton (1998), a fauna ictiológica desenvolve inúmeras estratégias de reprodução, coincidindo com períodos de maior diposição de alimento e abrigo para sua prole (observado no presente estudo), ao ser demonstrado que o período com entrada de água no sistema foi o que mais propiciou o desenvolvimento gonadal para a reprodução de A. pantaneiro.

No período de cheia foram capturados 19 indivíduos, sendo 9 em fase imatura. Neste período, a captura foi influenciada pela diluição dos indivíduos, devido a quantidade de água no sistema.
Os peixes desenvolvem diferentes estratégias, com diferentes funções vitais, mantendo-se presentes em épocas e locais com maior disponibilidade de abrigo e alimento (Wootton, 1998). Segundo Bonetto et al. (1981), a desova dos peixes destes sistemas de baías ocorre com mais intensidade no final do período da enchente, corroborando dados do presente estudo, cujo período de entrada de água no sistema representou a fase reprodutiva. Os períodos de vazante de água do sistema (menor profundidade da coluna d'água - estiagem), caracterizaram a fase de repouso dos peixes.

Os maiores valores para o índice gonadossomático do A. pantaneiro foram encontrados nos períodos de estiagem e enchente, indicando que esta espécie se reproduz, sobretudo, quando o sistema começa a receber água novamente (Figura 3).

Este fato pode ter ocorrido porque, no período de entrada de água no sistema, estes peixes iniciam o

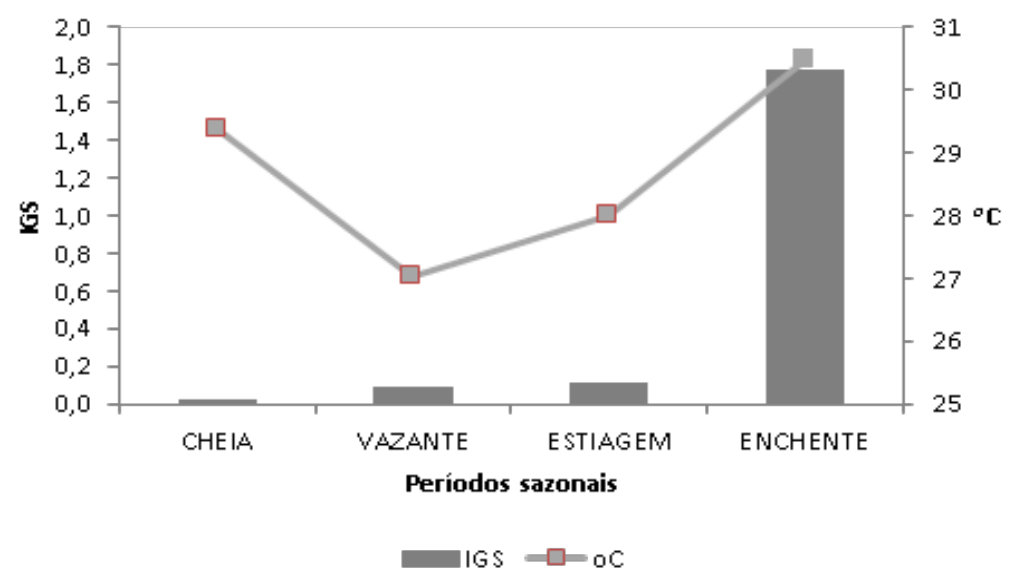

Figura 3 -Valores do índice gonadossomático de A. pantaneiro e da temperatura $\left({ }^{\circ} \mathrm{C}\right)$ da água no Sistema de baías Caiçara nos períodos de cheia, vazante, estiagem e enchente (2005/2007). 
período de maturação gonadal, elevando assim os valores deste índice. Os períodos de estiagem e enchente marcam o início do processo reprodutivo desta espécie neste sistema de baías, sendo mais evidente na enchente.

Assim, a reprodução desta espécie está associada às variações no ambiente aquático (temperatura e aumento das chuvas), adaptando o período reprodutivo de acordo com as condições favoráveis à sobrevivência de sua prole no ambiente (Vazzoler, 1996).

Os peixes desovadores de planície realizam pequenos movimentos transversais, afastando-se da planície de inundação rumo ao canal do rio, visando reproduzir na época das enchentes. 0 pulso de inundação é um processo fundamental à manutenção e conservação de rios com grandes planícies de inundação e suas espécies (Resende, 2008).

\section{Conclusão}

O pulso de inundação evidenciou importante fator determinante da reprodução de Acestrorhynchus pantaneiro no sistema de baía Caiçara; o início do processo reprodutivo se dá no período de enchente, mantendo-se em repouso nos períodos de vazante e estiagem.

\section{Agradecimentos}

A Universidade do Estado de Mato Grosso (UNEMAT) e ao Centro de Pesquisa em Limnologia, Biodiversidade e Etnobiologia do Pantanal (CELBE/UNEMAT) pela disponibilização de material de pesquisa e pela cessão da estrutura.

\section{Referências}

Agostinho AA, Gomes LC. Reservatório de Segredo: bases ecológicas para o manejo. Maringá: EDUEM; 1997.

Buckup PA, Menezes NA, Ghazzi MS. Catálogo das espécies de água doce do Brasil. Rio de Janeiro: Museu Nacional; 2007.

Bonetto AA, Canon Verón M, Roldán D. Nuevos aportes al conocimiento de las migraciones de peces en el Río Paraná. Ecosur. 1981; 8(16):29-40.
Brasil. Ministério do Interior. Estudos de Desenvolvimento Integrado da Bacia do Alto Paraguai (EDIBAP): Relatório da $1^{\text {a }}$ Fase, descrição física e recursos naturais. Brasília: SUDECO/EDIBAP; 1979.

Britski HA, Silimon KZS, Lopes BS. Peixes do Pantanal: manual de identificação de peixes do Pantanal. 2. ed. rev. ampl. Brasília: Embrapa Informação Tecnológica; 2007.

Da Silva CJ. Influência da variação do nível d'água sobre a estrutura e funcionamento de uma área alagável do Pantanal Mato-grossense (Pantanal Barão de Melgaço MT). 1990. 250 f. Tese (Doutorado em Ecologia e Recursos Naturais) Universidade Federal de São Carlos, São Paulo, 1990.

Silva CJ, Wantzen KM, Cunha CN, Machado FA. Biodiversity in the Pantanal Wetland, Brazil. In: Gopal B, Junk WJ, Davis JA. (Ed.). Biodiversity in wetlands: assessment, function and conservation. Leiden: Backhuys Publishers; 2001. p. 187-215.

Ferreira JCV. Mato Grosso e seus municípios. Cuiabá: Secretaria de Estado de Cultura; 1997.

Guerra TP, Floro RM, Melo VC, Aragão TV, Severi W. Período reprodutivo de Acestrorhynchus Britskii Menezes, 1969 no Reservatório de PA I, II, III, Rio São Francisco. In: Jornada de Ensino, Pesquisa e Extensão, 9., 2009, Recife. Anais... Recife: JEPEX, 2009.

Junk WJ, Da Silva C. O conceito de pulso de inundação e suas implicações para o Pantanal de Mato Grosso. In: Simpósio sobre Recursos Naturais e Socioeconômicos do Pantanal: manejo e conservação, 2., 1996. Corumbá. Anais... Corumbá: Embrapa Pantanal; 1996. p. 17-28.

Krinsk D. Dieta do peixe cachorro Acestrorhynchus pntaneiro Menezes, 1992 (Characidae: Acestrorhynchinae) do pantanal de Poconé, Mato Grosso, Brasil. Bioscience Journal. 2010; 26(2):287-295.

Menezes NA. Redefinição taxonômica das espécies de Acestrorhyncus do grupo Lacustris com a descrição de uma nova espécie (Osteichthyes, Characiformes, Characidae). Comunicações do Museu de Ciências PUCRS. 1992; 5(5):39-54.

Muniz CC. Avaliação do papel do pulso de inundação sobre a riqueza e biodiversidade de peixes em ambiente inundável, no Sistema de Baías Caiçara, Porção Norte do Pantanal mato-grossense, Alto Paraguai. 2010. 84 f. Tese (Doutorado em Ecologia e Recursos Naturais) - Centro de Ciências Biológicas e da Saúde, Universidade Federal de São Carlos, São Paulo, 2010. 
Nogueira FMB, Oliveira RD. Characterization of the fishes and of subsistence fishing in the Pantanal of Mato Grosso, Brazil. Revista Brasileira de Biologia. 2000; 60(3):435-445. doi:10.1590/S0034-71082000000300008.

Novaes JLC, Caramaschi P, Winemiller KO. Feeding of Cichla monoculus Spix, 1829 (Teleostei: Cichlidae) during ande after reservoir formation in the Tocantins River, Central Brazil. Acta Limnologica Brasiliensia. 2004; 16(1):41-49.

Paiva MP. Crescimento, reprodução e alimentação da traíra Hoplias malabaricus (Bloch), no nordeste brasileiro. Fortaleza: Imprensa Universitária do Ceará; 1974.

Resende EK. Pulso de inundação: processo ecológico essencial à vida no Pantanal [recurso eletrônico]. Corumbá: Embrapa Pantanal; 2008.

Ribeiro CS, Moreira RG. Fatores ambientais e reprodução de peixes. Revista da Biologia. 2012; 8:58-61.

Rodríguez M, Lewis WMJr. Regulation and stability in fish assemblages of neotropical gradients in lakes. Oecologia. 1994; 99:166-180.

Saccol-Pereira A, Milani PCC, Fialho CB. primeiro registro do Acestrorhynchus pantaneiro Menezes, 1992 (Characiformes, Acestrorynchicidae) no sistema da laguna dos patos, Rio Grande do Sul, Brasil. Biota Neotropica. 2006; 6(3):1-4.
Vazzoler AEAM. Biologia da reprodução de peixes teleósteos: teoria e prática. Maringá: EDUEM; 1996.

Vianello RL, Alves AR. Meteorologia básica e aplicações. Viçosa: Editora UFV, 2000.

Wootton RJ, Evans GW, Mills L. Annual cycle in female Threespined sticklebacks (Gasterosteus aculeatus L.) from an upland and lowland population. Journal of Fish Biology. 1978; 12(4):331-343. doi:10.1111/j.1095-8649.1978.tb04178.x.

Wootton RJ. Introduction: strategies and tacties in fish reproduction. In: Potts GW, Wootton RJ. (Eds.). Fish reproduction: strategies and tactics. London: Academic Press; 1984. p. 1-12.

Wootton R. Ecology of Teleost Fishes. Berlin: Springer; 1998.

Zaniboni-Filho E, Meurer S, Shibatta AO, Nuñer APO. Catálogo ilustrado de peixes do Alto Rio Uruguai. Florianópolis: Editora da UFSC; 2004.

Recebido em: 03/02/2014

Received in: 02/03/2014

Aprovado em: 14/10/2015

Approved in: 10/14/2015 